\title{
AC Losses in Non-inductive SFCL Solenoidal Coils Wound by Parallel Conductors
}

\author{
Wenjuan Song, Member, IEEE, Xiaoze Pei, Xianwu Zeng, Mohammad Yazdani-Asrami, Member, \\ IEEE, Xinyu Fang, Jin Fang, and Zhenan Jiang, Member, IEEE
}

\begin{abstract}
Large scale resistive superconducting fault current limiters (SFCLs) have attracted great interest in electric power systems and aviation applications due to their compactness, lightweight, automatic fault current limiting and fast recovery characteristics. Non-inductive coil wound with stacked conductors in parallel connection is commonly used to achieve high current rating for SFCL. It is of great significance and engineering value to minimize AC losses in non-inductive coil by optimizing the configuration of the stacked conductors in order to reduce the thermal load for cooling system. In this paper, multiple possible configurations of non-inductive stacks where each turn is composed of one, two and three conductors, respectively, were proposed. Numerical models were developed to investigate the effect of non-inductive stack configuration on AC loss. 8-turn noninductive solenoidal coils wound with the non-inductive stacks mentioned in the above were also simulated. It is concluded that the configuration of the non-inductive stack significantly affect the perpendicular magnetic field distribution in the end edges of the stack. Single layer configurations have limited magnetic field cancellation effect. The stack configuration with low AC loss and simple structure was suggested.
\end{abstract}

Index Terms-Superconducting fault current limiter, AC loss, non-inductive solenoidal coil, non-inductive stack, configuration.

\section{INTRODUCTION}

$\mathrm{F}$ AULT current limiting function of high temperature superconductors (HTS) has attracted great attention for electrical applications, to cope with the high fault current (normally 10-30 times nominal current) beyond the capability of circuit breaker [1]-[9]. Resistive type superconductor fault current limiters (SFCLs) exhibit advantages over other traditional fault current limiting devices: automatic fault current limitation, fast recovery, simple structure, compact size, lightweight, and fail safe [10]-[11]. Therefore, resistive SFCLs are favorable component in electric systems to protect the power system and maintain a high degree of safety standard [12]-[13].

There are three typical SFCL coil topologies: pancake type, straight line type, and solenoidal type. Although solenoidal

This work is funded by the UK EPSRC, Developing Superconducting Fault Current Limiters (SFCLs) for Distributed Electric Propulsion Aircraft: EP/S000720/1.

W. Song and X. Pei are with the Department of Electronic \& Electrical Engineering, The University of Bath, Bath, BA2 7AY, U.K. (e-mails: ws603@bath.ac.uk, x.pei@bath.ac.uk).

$\mathrm{X}$. Zeng is with the Department of Mechanical Engineering, The University of Bath, Bath, BA2 7AY, U.K. (e-mail: xz2478@bath.ac.uk). non-inductive coils are less compact compared to pancake type non-inductive coils, they are favorable in dissipating tremendous fault energy effectively in a short time to protect the SFCL windings from burning out [14]-[16], and distributing mechanical force over larger area during the fault to reduce the stress imposed on windings. In addition, straight line type coil fails to achieve the compactness of SFCL.

Large scale resistive type SFCLs require high current carrying capacity and low AC loss which could be achieved using non-inductive coil structure [17]. There are several candidates for high current carrying capacity: conductor on round core cables (CORCs) [18]-[19], Roebel cables [20]-[21], and vertical stacks where multiple superconductors were connected in parallel [22]-[24]. However it is difficult to wind non-inductive coils using CORC cables due to its round shape. Vertical stacks are more cost-effective and easier to be manufactured as compared to Roebel cables. Thus, noninductive coil wound by simple stacks is commonly implemented to obtain high current carrying ability of SFCLs.

To date, some SFCL projects have successfully demonstrated their pancake type non-inductive SFCL coils wound with stacks for high current ratings [25]-[28], and low AC loss was achieved by specific configuration of conductors in noninductive stacks. However, there is lack of reports for solenoidal non-inductive SFCL coils wound with stacks, and it is critically needed to address the effect of non-inductive stack configuration on AC loss in solenoidal SFCL coils.

Non-inductive coil wound by single conductor is called bifilar coil, and non-inductive coil wound by stack with more than two conductors is called multifilar coil. In this work, noninductive stacks where each turn is wound with one, two and three conductors were considered, for solenoidal non-inductive SFCL coils. The AC loss values in non-inductive stacks with different configurations were simulated and analyzed, using numerical calculations in 2D finite element model (FEM). Magnetic field distributions for various configurations of noninductive stacks were studied and compared. AC loss behavior

M. Yazdani-Asrami, is with Department of Electronic and Electrical Engineering, University of Strathclyde, Glasgow, G1 1XW, UK (e-mail: mohammad.yazdani-asrami@strath.ac.uk).

X. Fang and J. Fang are with School of Electrical Engineering, Beijing Jiaotong University, China. (e-mail:19117005@bjtu.edu.cn,jfang@bjtu.edu.cn) Z. Jiang is with Robinson Research Institute, Victoria University of Wellington, New Zealand. (e-mail: zhenan.jiang@vuw.ac.nz) 
TABLE I

DIFFERENT CONFIGURATIONS OF NON-INDUCTIVE STACKS WOUND OF ONE, TWO AND THREE CONDUCTORS IN PARALLEL

\begin{tabular}{|c|c|c|c|}
\hline \multirow{3}{*}{$\begin{array}{l}\text { No. of layer in one } \\
\text { stack }\end{array}$} & \multicolumn{3}{|c|}{ Naming of each non-inductive stack } \\
\hline & BFS & MFS4 & MFS6 \\
\hline & $\mathrm{N}_{\mathrm{p}, \text { each turn }}=1$ & $\mathrm{~N}_{\mathrm{p} \text {, each turn }}=2$ & $\mathrm{~N}_{\mathrm{p}, \text { each turn }}=3$ \\
\hline$N_{\text {layer }}=1$ & AB_v & ABAB_vv & ABABAB_vv \\
\hline & & AABB_vv & AAABBB_vv \\
\hline$N_{\text {layer }}=2$ & $\mathrm{AB} \_\mathrm{h}$ & $\begin{array}{l}\text { AABB_vh } \\
\text { AABB_hv } \\
\text { ABBA_vh }\end{array}$ & AAABBB_vh \\
\hline$N_{\text {layer }}=3$ & & & $\begin{array}{l}\text { AAABBB_hv } \\
\text { ABBAAB_vh }\end{array}$ \\
\hline$N_{\text {layer }}=4$ & & $\begin{array}{l}\text { AABB_hh } \\
\text { ABAB_hh }\end{array}$ & \\
\hline$N_{\text {layer }}=6$ & & & $\begin{array}{l}\text { AAABBB_hh } \\
\text { ABABAB hh }\end{array}$ \\
\hline
\end{tabular}

Note: $\mathrm{v}$ and $\mathrm{h}$ represents vertical and horizontal layout, respectively. $\mathrm{AB} \mathrm{v}$ indicates the bifilar stack where the two conductors are assembled vertically "head to head (HTH)", whilst carrying current in opposite directions. AB_h means the two conductors are assembled horizontally "side by side (SBS)" whilst carrying current in opposite directions. Take AABB_vh as an exmple, $v$ means conductors in $\mathrm{AA}$ and $\mathrm{BB}$ are both arranged vertically, while conductor bundle of AA and BB are assembled horizontally."

in 8-turn non-inductive solenoidal coils wound with the above non-inductive stacks were investigated and discussed.

\section{StACK CONFIGURATIONS}

Table I tabulates possible configurations of non-inductive stacks where each turn is composed of one, two and three parallel connected conductors, categorized by the number of horizontal layer $N_{\text {layer }}$ in one stack. The more layer number, the more complex coil structure and its manufacturing. These stacks are classified as BFS (bifilar stack where each turn is composed of one conductor only), MFS4 (multifilar stack where each turn is composed of two conductors), and MFS6 (multifilar stack where each turn is composed of three conductors), based on the conductor number in each turn. The schematics for these configurations are shown in Figs. 1-3. The spacing between vertical conductors is $2 \mathrm{~mm}$, and the spacing between horizontal conductors is assumed as $2 \mathrm{~mm}$.

There are two configurations for $\mathrm{BFS}, \mathrm{AB} \mathrm{v}$ and $\mathrm{AB} h$, as shown in Fig. 1(a) and 1(c). Fig. 1(b) and Fig. 1(d) plot the 3D view of these two configurations. AB_v denotes the bifilar stack where the two conductors are assembled vertically "head to head (HTH)", whilst carrying current in opposite directions. $\mathrm{AB} \_\mathrm{h}$ denotes the bifilar stack where the two conductors are assembled horizontally "side by side (SBS)" whilst carrying current in opposite directions. The conductor marked with red outline denotes the outermost conductor in the stack, and it applies for all the stacks studied in the paper. The red arrow guides the direction from the top to the bottom of the crosssection along conductor width, and this direction helps to show the magnetic field distribution in section IV. There are seven possible configurations for MFS4, arranged in one, two and four layers, as shown in Fig. 2(a) - (g). Fig. 2(i) plots the 3D view of stack AABB vh for a better understanding. Similarly, Fig. 3(a)-(g) show seven possible configurations for MFS6, arranged in one, two, three and six layers.

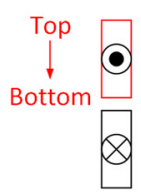

(a) 2D: $A B \_v$

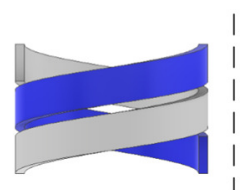

(b) 3D: AB_v

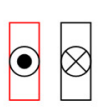

(c) $2 \mathrm{D}: \mathrm{AB}_{-} \mathrm{h}$

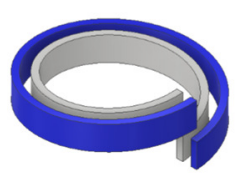

(d) 3D: AB_h

Fig. 1. Configurations of bifilar stacks composed of two superconductors (BFS). (a) 2D diagram of $\mathrm{AB}$-v. (b) 3D diagram of the solenoidal coil wound by $\mathrm{AB}$-v.. (c) $2 \mathrm{D}$ diagram of $\mathrm{AB} \_\mathrm{h}$. (d) $3 \mathrm{D}$ diagram of the solenoidal coil wound by $\mathrm{AB}_{-} \mathrm{h}$. The conductor with red outline denotes the outermost conductor in the stack. The red arrow directs the top to the bottom in conductor cross-section (i.e, one side to another side along conductor length). This orientation applies for all the stacks in this paper.

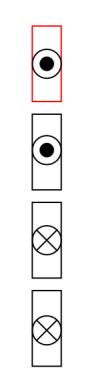

(a)

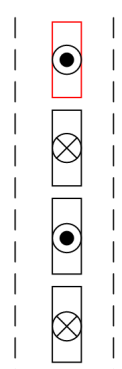

(b)

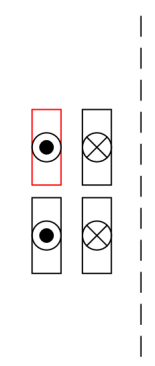

(c)

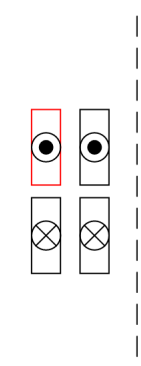

(d)

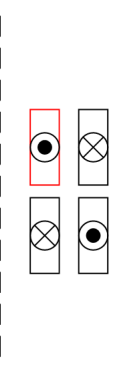

(e)

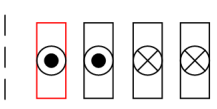

(f)

AABB_hh
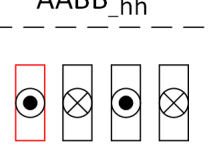

(g)
$A A B B_{-} v v$ ABAB_vv $A A B B_{-} v h \quad A A B B_{-} h v \quad A B B A_{-} v h$

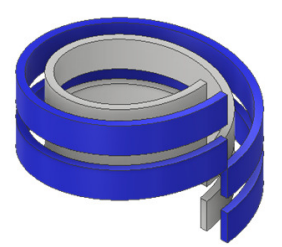

(i) 3D view for AABB_vh

Fig. 2. (a)-(g) Seven configurations (a)-(g) of multifilar stacks composed of four superconductors (MFS4). (i) 3D view of on turn AABB_vh, as an example. The conductor with red outline denotes the outermost conductor in the stack.

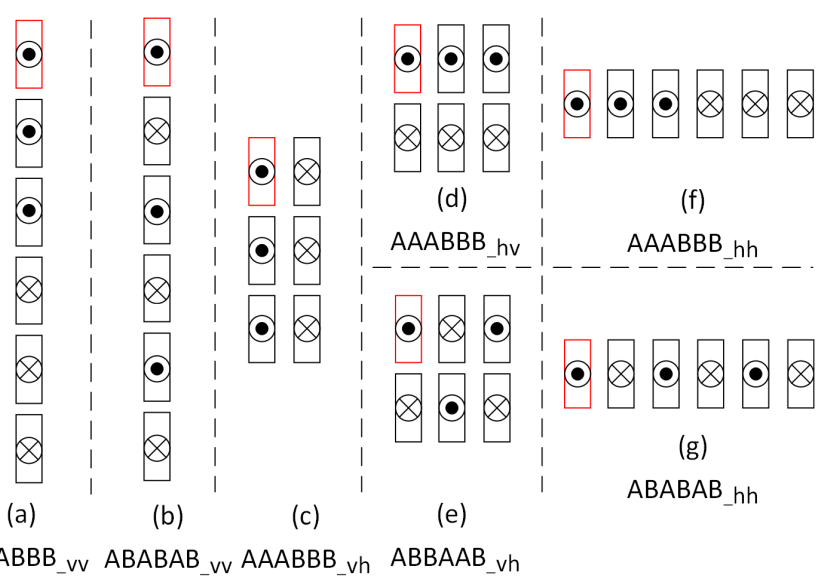

Fig. 3. Configurations of multifilar stacks composed of six superconductors (MFS6). The conductor with red outline shows the outermost tape in the stack. 
TABLE II

\begin{tabular}{lc}
\multicolumn{2}{c}{ PARAMETERS OF HTS COATED CONDUCTOR } \\
\hline \hline Manufacturer & SuperPower \\
Conductor width, mm & 4 \\
Conductor thickness, mm & 0.1 \\
Thickness of superconducting layer, $\mu \mathrm{m}$ & 1 \\
Stabilizer material & Copper \\
Thickness of stabilizer, $\mu \mathrm{m}$ & 20 \\
Substrate material & Hastelloy \\
Thickness of substrate, $\mu \mathrm{m}$ & 50 \\
Critical current of superconductor $@ 77 \mathrm{~K}$, self-field, $I_{\mathrm{c}}(\mathrm{A})$ & 86 \\
\hline \hline
\end{tabular}

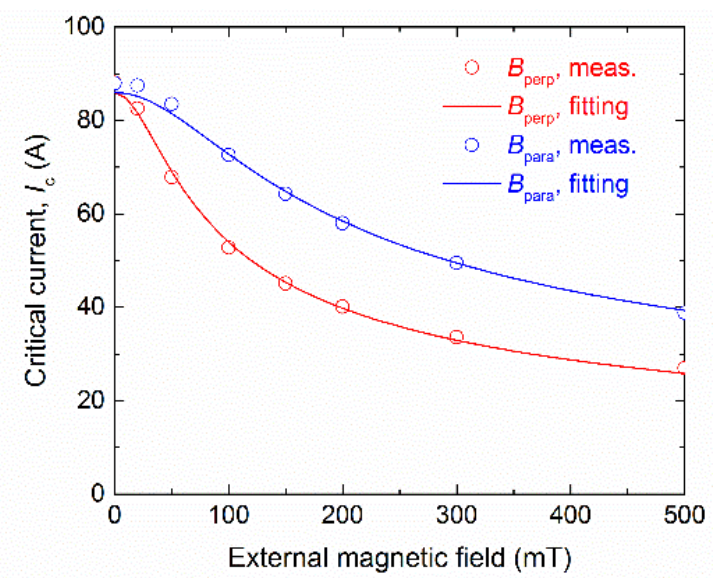

Fig. 4. Critical current dependence on external magnetic field.

\section{Modelling Method AND SETUP}

\section{A. Modelling of multifilar stacks}

The superconductor used to wind the non-inductive stack and non-inductive coil is $4 \mathrm{~mm}$-wide YBCO coated conductor from SuperPower, the same conductor as used in our previous research [29]. Since AC losses in non-inductive coils are the studied target, only superconducting layer was simulated. The detailed parameters are listed in Table II.

Numerical calculation for non-inductive stacks were carried out by 2D FEM models using COMSOL Multiphysics, by means of $H$ formulation [30]-[37]. Two independent variables were implemented in the model, $\boldsymbol{H}=\left[H_{x}, H_{\mathrm{y}}\right]^{\mathrm{T}}$, where $H_{\mathrm{x}}$ and $H_{\mathrm{y}}$ are parallel and perpendicular magnetic fields. $E-J$ power law was used to represent the highly nonlinear relationship of local electric field $E$ and local current density $J$ in the HTS layer:

$$
E / J=\left(E_{0} / J_{c}(B, x)\right)\left(\left|J / J_{c}(B, x)\right|\right)^{n-1}
$$

where $E_{0}=1 \mu \mathrm{V} / \mathrm{cm}, n=30$ is the power index derived from $V$ $I$ characteristic, and $J_{\mathrm{c}}(\boldsymbol{B}, x)$ is the critical current density dependence on local magnetic field and lateral position, and it is expressed in (2). Here, $J_{c}(x)$ is critical current density dependence on lateral position [38]-[39]. A modified Kim model was adopted to show the effect of magnetic field on local critical current density [40]-[41]. $k=0.41, \alpha=0.24$, and $B_{0}=$ $41 \mathrm{mT}$ are curve fitting parameters [29].

$$
J_{\mathrm{c}}(\boldsymbol{B}, x)=J_{\mathrm{c}}(x)\left(1+\left(k^{2} B_{\|}^{2}+B_{\perp}^{2}\right) / B_{0}^{2}\right)^{-\alpha}
$$

Fig. 4 plots and compares the measured $I_{\mathrm{c}}(\boldsymbol{B}, x)$ performance and the fitted curves.

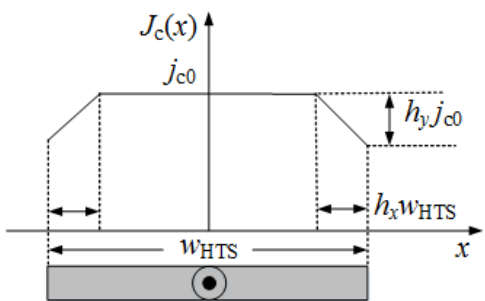

Fig. 5. Schematic of lateral distribution of critical current density, $J_{\mathrm{c}}(x)$ along tape width.

A trapezoidal lateral distribution of critical current, $J_{\mathrm{c}}(x)$ was assumed in calculation [29]. Fig. 5 gives the schematic of $J_{\mathrm{c}}(x)$ distribution along tape width, $w_{\text {HTS, }}$ which is mathematically expressed as (3), assuming critical current density is reduced symmetrically toward tape edges. The non-uniform characteristic is shown by two parameters: $h_{\mathrm{x}}$ defines the tape width portion with reduced critical current density at each edge; and $h_{\mathrm{y}}$ represents the portion of reduced critical current density at the tape edge compared to the critical current density at the tape center, $j_{\mathrm{c} 0}$. The parameters used in this work are $h_{\mathrm{x}}=15 \%$ and $h_{\mathrm{y}}=60 \%$.

$J_{\mathrm{c}}(x)=j_{\mathrm{c} 0}\left\{\begin{array}{cl}1 & \text { if }|x| \leq \frac{1-h_{x}}{2} w_{\mathrm{HTS}} \\ 1-h_{y}-\left(\frac{|x|}{w_{\mathrm{HTS}}}-\frac{1}{2}\right) \frac{h_{y}}{h_{x}} & \text { if }|x|>\frac{1-h_{x}}{2} w_{\mathrm{HTS}}\end{array}\right.$

The $j_{\mathrm{c} 0}$ can be calculated from the measured self-field critical current, $I_{\mathrm{c} 0}$, as shown in (4),

$$
j_{\mathrm{c} 0}=\frac{I_{\mathrm{c} 0}}{w_{\mathrm{HTS}} h_{\mathrm{HTS}}\left(1-h_{x} h_{y}\right)}
$$

The governing equation is derived and expressed in (5) combining Faraday's law, Ampere's law, Ohm's law, and constitutive law [30]-[37], [42]. Equation (6) can be obtained by substituting two variables $H_{x}$ and $H_{y}$ into (5).

$$
\begin{gathered}
\partial\left(\mu_{0} \mu_{r} \boldsymbol{H}\right) / \partial \mathrm{t}+\nabla \times(\rho \nabla \times \boldsymbol{H})=0 \\
\mu_{0} \mu_{r} \frac{\partial H_{x}}{\partial t}-\frac{\partial\left(\rho\left(\frac{\partial H_{y}}{\partial x}-\frac{\partial H_{x}}{\partial y}\right)\right)}{\partial y}=0 \\
\mu_{0} \mu_{r} \frac{\partial H_{y}}{\partial t}+\frac{\partial\left(\rho\left(\frac{\partial H_{y}}{\partial x}-\frac{\partial H_{x}}{\partial y}\right)\right)}{\partial x}=0
\end{gathered}
$$

\section{B. Modelling of solenoidal coils}

For simplification, 2D axisymmetric model was employed to simulate the electromagnetic behavior in solenoidal coils, with the two independent variables of $\boldsymbol{H}=\left[H_{r}, H_{z}\right]^{\mathrm{T}}$. The governing equations can be expressed in (7) [43]-[45],

$$
\left\{\begin{array}{c}
\mu_{0} \mu_{r} \frac{\partial H_{r}}{\partial t}-\frac{1}{r} \frac{\partial\left(r \rho\left(\frac{\partial H_{r}}{\partial z}-\frac{\partial H_{z}}{\partial r}\right)\right)}{\partial z}=0 \\
\mu_{0} \mu_{r} \frac{\partial H_{z}}{\partial t}+\frac{1}{r} \frac{\partial\left(r \rho\left(\frac{\partial H_{r}}{\partial z}-\frac{\partial H_{z}}{\partial r}\right)\right)}{\partial r}=0
\end{array}\right.
$$




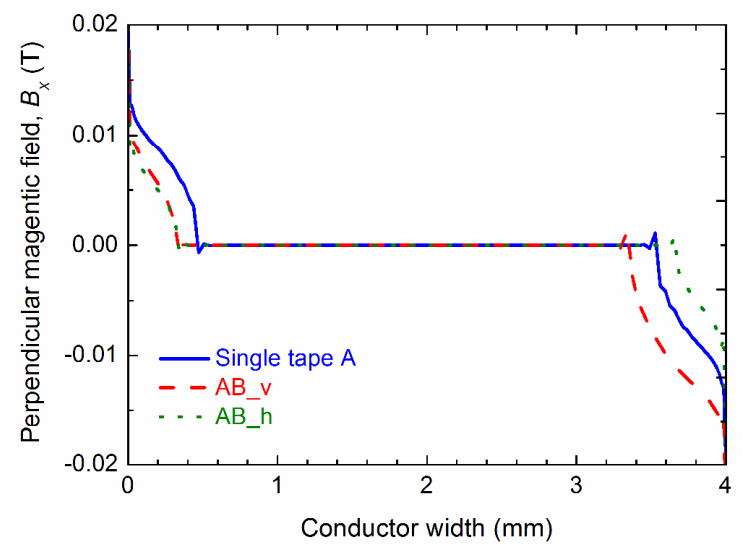

Fig. 6. Perpendicular magnetic field distribution, $B_{\mathrm{x}}$, along the width of the outermost superconductor in bifilar stack.

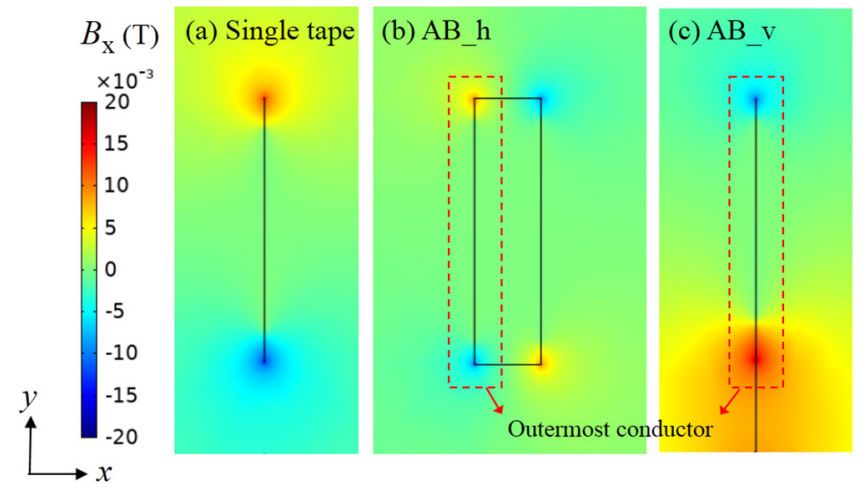

Fig. 7. Perpendicular magnetic field distribution in outermost conductor of BFS and single tape, at $I_{\mathrm{t}, \text { peak }} / I_{\mathrm{c} 0}=0.5$ and $t=3 / 4 T$.

\section{STACK AND COIL AC Loss ANALYSIS}

\section{A. AC loss in non-inductive stacks of BFS}

Fig. 6 and Fig. 7 plot the perpendicular magnetic field distribution, $B_{x}$, along the width of the outermost conductor and around it in bifilar stacks and single tape, at $t=3 / 4 T$, where $T$ is the period of excitation current. As mentioned, the outermost conductor was marked by red outline in Fig. 1. The $\mathrm{x}$-axis is conductor width, from 0 to $4 \mathrm{~mm}$, orientated from the top to the bottom of the tape.

As observed in Fig. 6, the penetration depth, $\lambda$, of outermost tape in AB_h is the lowest in top and bottom section, due to best magnetic field cancellation effect, shown in Fig. 7(b). At the bottom section, the penetration depth of outermost tape in $A B \_v$ is bigger than that in single tape, due to that the perpendicular magnetic field was strengthened rather than weakened in this area using this configuration, as shown in Fig. 7(c). However, at the top section, the penetration depth of outermost tape in $\mathrm{AB}_{-} \mathrm{v}$ is smaller than that in single tape, indicating that perpendicular magnetic field was cancelled to some extent using this configuration.

Fig. 8 shows the calculated AC losses in bifilar stack composed of two conductors (BFS) and a single tape, and compares the simulation results with Norris-ellipse (N-e) and Norris-strip (N-s) models [46]. AC losses in the single tape fall between $\mathrm{N}-\mathrm{e}$ and $\mathrm{N}$-s theoretical curves, but closer to N-e curve.

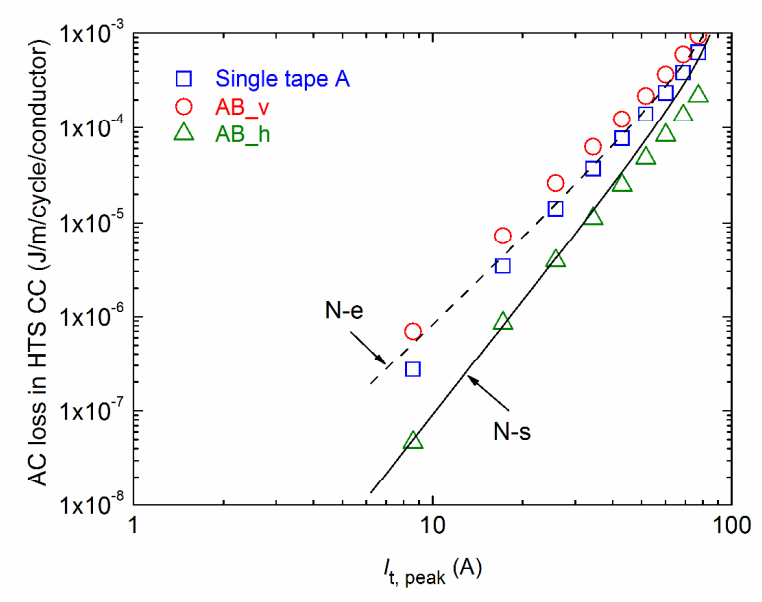

Fig. 8. Calculated AC loss in bifilar stack at $54 \mathrm{~Hz}$.

This can be explained by the non-uniform $J_{\mathrm{c}}$ distribution along the width of the tape caused during slitting process [47].

$\mathrm{AC}$ loss in bifilar stacks increases as the transport current increase. $\mathrm{AC}$ losses in $\mathrm{AB} \mathrm{h}, \mathrm{AB} \_\mathrm{v}$ and single tape, denoted as $Q_{\mathrm{AB}_{-} \mathrm{h}}, Q_{\mathrm{AB}_{-} \mathrm{v}}$ and $Q_{\text {single }}$, follow the tendency of $Q_{\mathrm{AB}_{-} \mathrm{v}}>Q_{\text {single }}>$ $Q_{\mathrm{AB} \_ \text {h, }}$ at any transport current. This is consistent with the magnetic field distribution around $\mathrm{AB}_{-} \mathrm{h}, \mathrm{AB}_{-} \mathrm{v}$ and single tape. At $I_{\text {t, peak }}=25.8 \mathrm{~A}\left(I_{\mathrm{t} \text {, peak }} / I_{\mathrm{c} 0}=0.3\right), Q_{\mathrm{AB} \_\mathrm{h}}$ accounts for only $15.1 \%$ of $Q_{\mathrm{AB}_{-} \mathrm{v}}$ and $27.9 \%$ of $Q_{\text {single. }}$ At $I_{\mathrm{t} \text {, peak }}=60.2 \mathrm{~A}\left(I_{\mathrm{t} \text {, peak }} / I_{\mathrm{c} 0}=0.7\right)$, $Q_{\mathrm{AB}_{-} \mathrm{h}}$ accounts for $22.7 \%$ of $Q_{\mathrm{AB}_{-} \mathrm{v}}$ and $35.3 \%$ of $Q_{\text {single }}$.

\section{B. AC loss in non-inductive stacks of MFS4}

Fig. 9 and Fig. 10 show perpendicular magnetic field distribution, $B_{\mathrm{x}}$, along the width of the outermost superconductor and around it in MFS4. Fig. 9(a) plots the overall view of $B_{\mathrm{x}}$ distribution, indicating that $B_{\mathrm{x}}$ distributes asymmetrically at top and bottom edges as shown in Fig. 9. $B_{\mathrm{x}}$ distribution changes drastically at the bottom edges in different stack configurations. The maximum $B_{\mathrm{x}}$ in the bottom edges varies from $10 \mathrm{mT}$ to $25 \mathrm{mT}$ among all stack configurations. Fig. 9(b) shows the enlarged $B_{\mathrm{x}}$ distribution in the top edges of MFS4 for seven configurations. It is observed that single tape and the outermost conductor in AABB_vv has the deepest penetration depth up to $0.51 \mathrm{~mm}$. The penetration depth for other stacks deviates between 0.37 to $0.41 \mathrm{~mm}$. Fig. 9(c) shows the enlarged $B_{\mathrm{x}}$ distribution in the edges of MFS4 for seven different configurations. AABB_hv has the deepest penetration depth, up to $0.87 \mathrm{~mm}$, owing to the highest magnetic field shown in Fig. 10(e). Magnetic field penetration depths are the smallest for the stacks ABAB_hh, AABB_vh and ABBA_vh, sharing a similar value of $0.37 \mathrm{~mm}$, due to cancellation of the perpendicular magnetic field components with the neighboring conductors, shown in Figs. 10(g), 10(d) and 10(f).

Fig. 11 shows the AC losses in multifilar stacks composed of four conductors (MFS4) with seven different configurations, plotted together with the AC loss in single tape and Norris models. The frequency for AC loss calculation is $54 \mathrm{~Hz}$. AC loss values in the stacks, $Q_{\mathrm{ABAB}_{-} \mathrm{vv}}, Q_{\mathrm{AABB}_{-} \mathrm{vv}}, Q_{\mathrm{AABB}_{\mathrm{V}} \mathrm{vh}}, Q_{\mathrm{AABB} \_\mathrm{hv}}$,

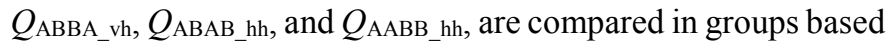
on layer number of the stack. 


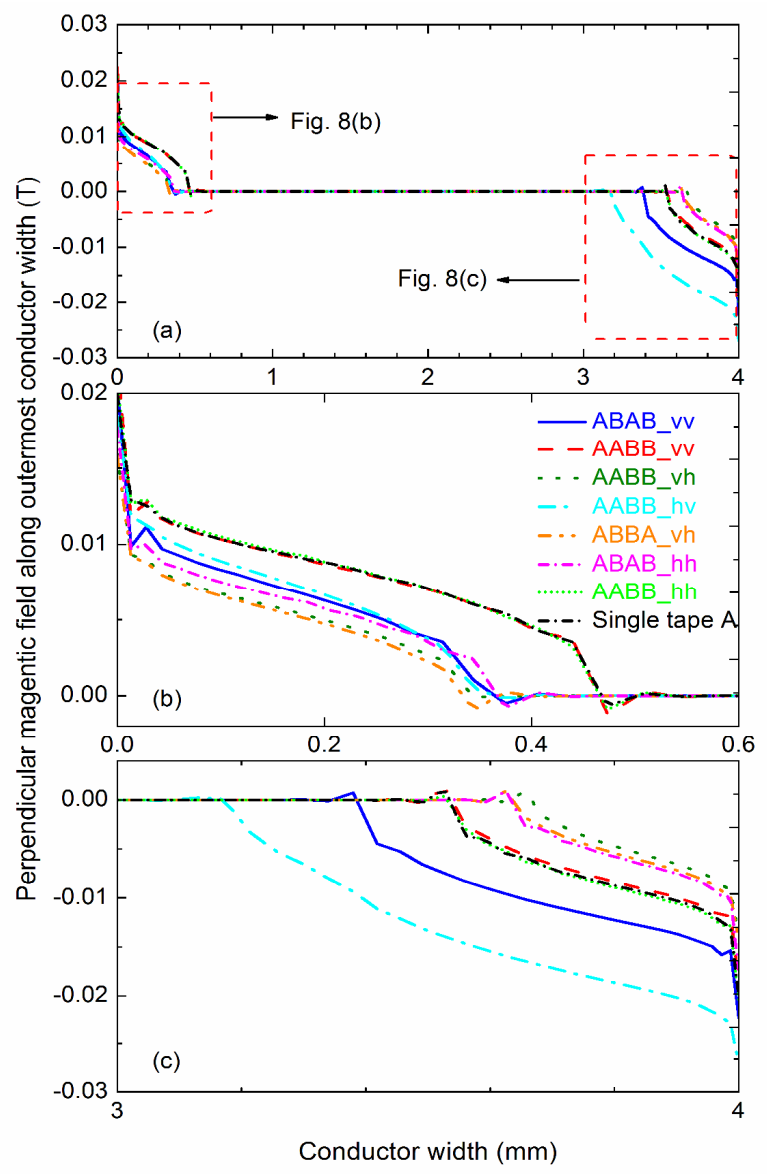

Fig. 9. Perpendicular magnetic field distribution, $B_{x}$, along the width of the outermost conductor in multifilar stack composed of four conductors, MFS4. (a) Overall view of $B_{x}$ distribution. (b) Enlarged $B_{x}$ distribution in top section of outermost conductor. (c) Enlarged $B_{\mathrm{x}}$ distribution in bottom section of outermost conductor.

1) At first we compare AC loss values in the one-layer MFS4, i.e. $\mathrm{ABAB}_{-} \mathrm{vv}$ and $\mathrm{AABB} \_\mathrm{vv}$. AC loss values follow $Q_{\text {single }}$ $<Q_{\mathrm{ABAB}_{-} \mathrm{vv}}<Q_{\mathrm{AABB}_{-} \mathrm{vv}}$, at any current amplitude. In other words, AC loss in the MFS4 with only one layer exceeds $Q_{\text {single }}$, due to strengthening of perpendicular magnetic field components in some of the gaps. The largest superposition of perpendicular magnetic field components occurs in the middle gap between the two upper conductors and the two lower conductors. At At $I_{\mathrm{t} \text {, peak }}=43 \mathrm{~A}\left(I_{\mathrm{t} \text {, peak }} / I_{\mathrm{c} 0}=0.5\right), Q_{\mathrm{ABAB}_{-} \mathrm{vv}}$ is $68.4 \%$ of $Q_{\mathrm{AABB}_{-} \mathrm{vv}}$ and $144.6 \%$ of $Q_{\text {single. }}$

2) When the conductors of MFS4 are configured in two layers, AC losses follows $Q_{\text {AABB_vh }}<Q_{\text {ABBA_vh }_{-}}<Q_{\text {single }}<$ $Q_{\mathrm{AABB} \_ \text {hv. }} Q_{\mathrm{AABB}_{-} \mathrm{vh}}$ is slightly smaller than $Q_{\mathrm{ABBA}_{\text {_vh }}}$. At $I_{\mathrm{t} \text {, peak }}=$ $43 \mathrm{~A}\left(I_{\mathrm{t}, \text { peak }} / I_{\mathrm{c} 0}=0.5\right), Q_{\text {AABB_vh }}$ accounts for $91.8 \%$ of $Q_{\text {ABBA_vh }}$, $31.0 \%$ of $Q_{\text {single }}$, and $9.1 \%$ of $Q_{\text {AABB_hv. }}$.

3) When the conductors of MFS4 are configured in four layers side by side, $\mathrm{AC}$ losses follows $Q_{\mathrm{ABAB}_{-} \text {hh }}<Q_{\mathrm{AABB}_{-} \text {hh }}<$ $Q_{\text {single. }}$. At $I_{\mathrm{t} \text {, peak }}=43 \mathrm{~A}\left(I_{\mathrm{t} \text {, peak }} / I_{\mathrm{c} 0}=0.5\right), Q_{\mathrm{ABAB} \_ \text {hh }}$ accounts for $36.1 \%$ of $Q_{\text {AABB_hh }}, 31.0 \%$ of $Q_{\text {single }}$, and $28.0 \%$ of $Q_{\text {AABB_hv. }}$

Comparing the AC loss performance in the seven multifilar stacks, $Q_{\mathrm{ABAB} \_ \text {hh }}, Q_{\mathrm{AABB}_{-} v \mathrm{nh}}$ and $Q_{\mathrm{ABBA} \_ \text {vh }}$ agree well from one another, and also exhibit the lowest loss values in the seven stacks.

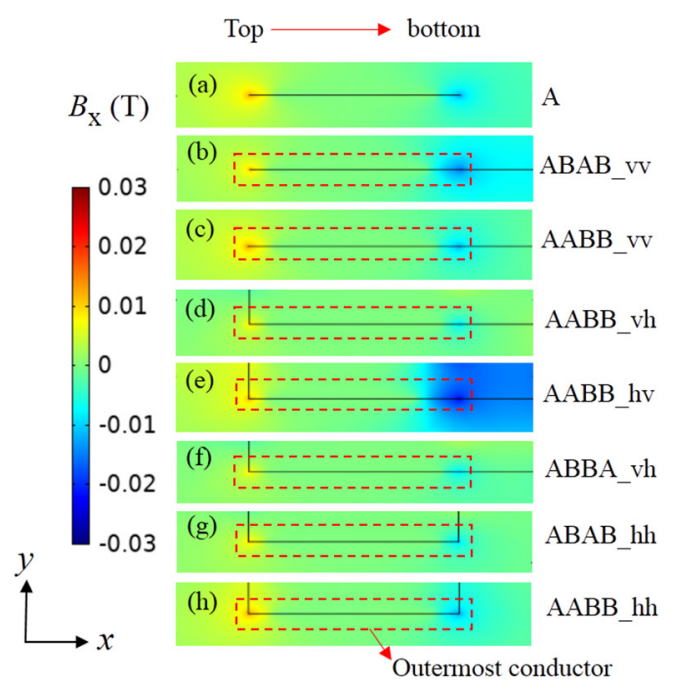

Fig. 10. Perpendicular magnetic field distribution in outermost conductor of MFS4 and single tape, at $I_{\mathrm{t}, \text { peak }} / I_{\mathrm{c} 0}=0.5$ and $t=3 / 4 T$.

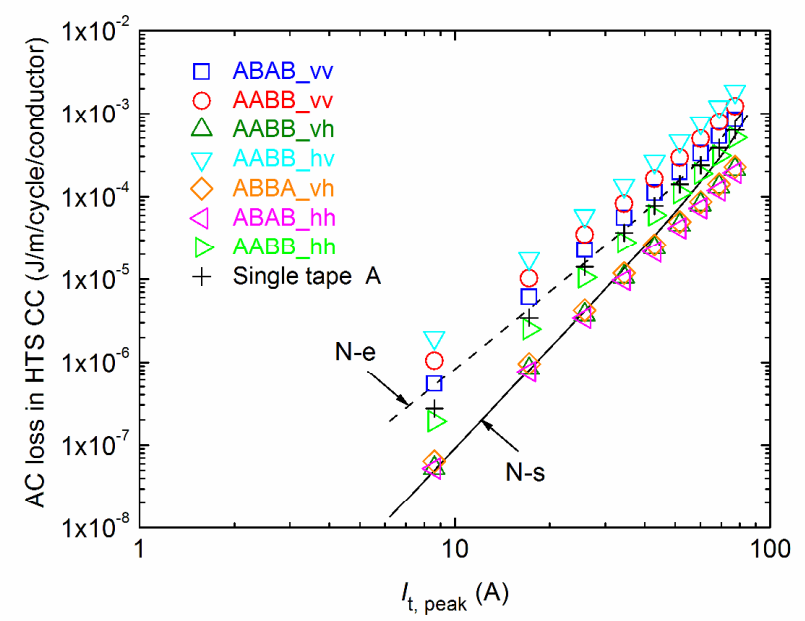

Fig. 11. Calculated AC losses in multifilar stacks composed of four conductors (MFS4) at $54 \mathrm{~Hz}$.

On the other hand, $Q_{\text {AABB_hv }}$ has the highest $\mathrm{AC}$ loss compared to the other stacks.

\section{AC loss in non-inductive stacks of MFS6}

Fig. 12 and Fig. 13 plot $B_{\mathrm{x}}$ distribution along the width of the outermost superconductor and around it in MFS6 for differing configurations. Fig. 12(a) shows the overall view of $B_{\mathrm{x}}$ distribution, indicating $B_{\mathrm{x}}$ allocates asymmetrically from top sections to bottom sections which agrees with Fig. 13, the same as $B_{\mathrm{x}}$ distribution in MFS4. There is a great deviation of $B_{\mathrm{x}}$ in bottom sections for different stack configuration.

Fig. 12(b) shows the enlarged $B_{\mathrm{x}}$ distribution in top sections of the outermost conductor in MFS6 for seven different configurations. The outermost conductor in AAABBB_vv has the deepest penetration depth in top section, due to the highest $B_{\mathrm{x}}$ value. The penetration depth for other stacks varies between $0.34 \mathrm{~mm}$ to $0.58 \mathrm{~mm}$. 


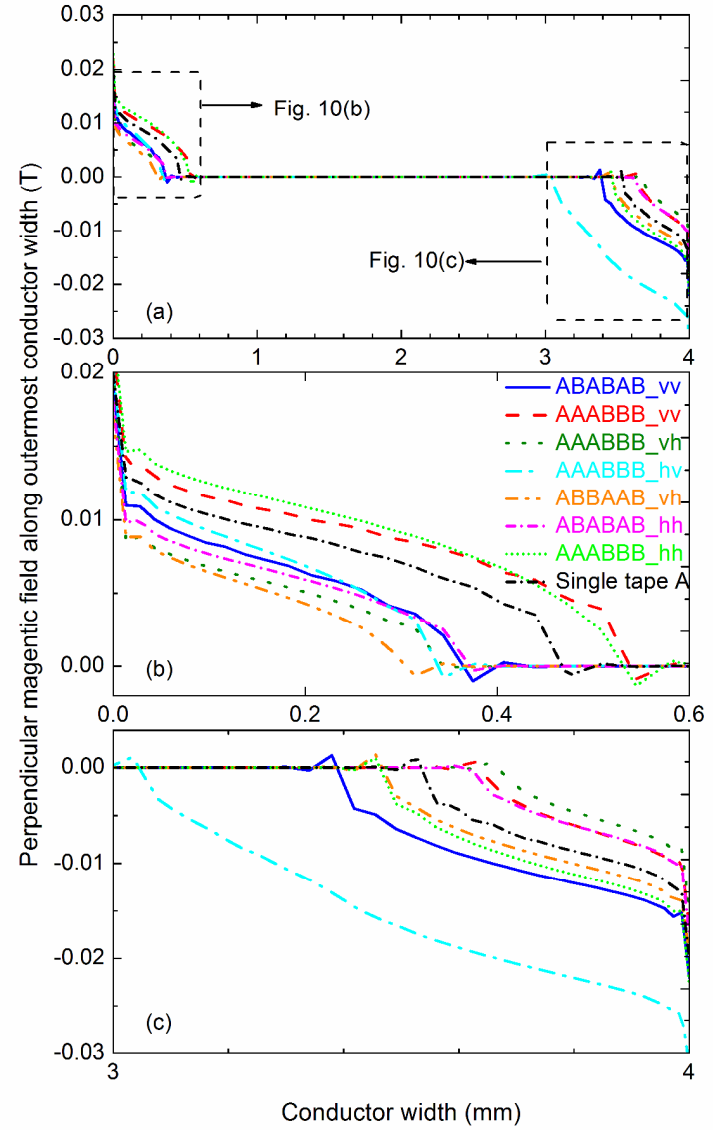

Fig. 12. Perpendicular magnetic field distribution, $B_{\mathrm{x}}$, along the width of the outermost conductor in multifilar stack composed of six conductors, MFS6. (a) Overall view of $B_{x}$ distribution. (b) Enlarged $B_{x}$ distribution in top section of outermost conductor. (c) Enlarged $B_{\mathrm{x}}$ distribution in bottom section of outermost conductor.

Fig. 12(c) shows an enlarged $B_{\mathrm{x}}$ distribution in bottom sections of the outermost conductor in MFS6 for seven configurations. AAABBB_hv has the deepest penetration depth driven by the magnetic field, up to $1.01 \mathrm{~mm}$. The penetration depth is the smallest for AAABBB_vh, $0.37 \mathrm{~mm}$, due to a lowest surrounded perpendicular magnetic field, indicating in Fig. 13(d). Besides, the penetration depth in ABABAB_hh is $0.41 \mathrm{~mm}$, slightly higher than AAABBB_vh.

The total penetration depth for AAABBB_vh and ABABAB_hh are $0.78 \mathrm{~mm}$ and $0.82 \mathrm{~mm}$, respectively, smaller than other stacks. Since the outermost conductor was chosen as a typical tape in the stack for magnetic field study, it provides a rough estimation for magnetic field distribution for each stack. $\mathrm{AC}$ loss dissipation in one stack is contributed by all the conductors. The highest penetration depth occurs in stack AAABBB_hv, and thus, the highest AC loss was expected and observed in Fig. 14.

Fig. 14 reports the AC losses in multifilar stacks composed of six conductors, MFS6, for seven different configurations, plotted with Norris models. AC loss in each stack, $Q_{\text {ABABAB_vv }}$, $Q_{\text {AAABBB_vv }}, Q_{\text {AAABBB_vh }}, Q_{\text {AAABBB_hv }}, Q_{\text {ABBAAB_vn }}, Q_{\text {ABABAB_hh }}$, and $Q_{\mathrm{AAABBB}}$ hh, , are compared in groups based on layer number of the stack.

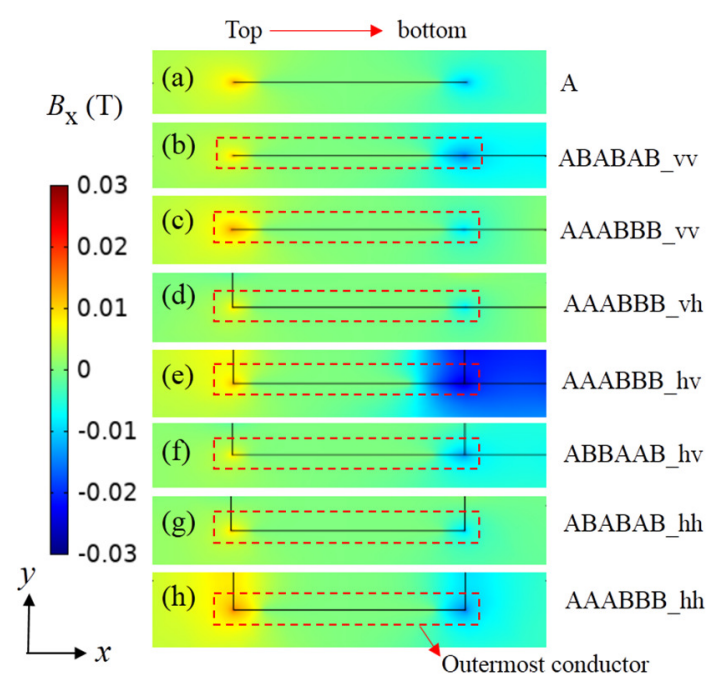

Fig. 13. Perpendicular magnetic field distribution in outermost conductor of MFS4 and single tape, at $I_{\mathrm{t}, \text { peak }} / I_{\mathrm{c} 0}=0.5$ and $t=3 / 4 T$.

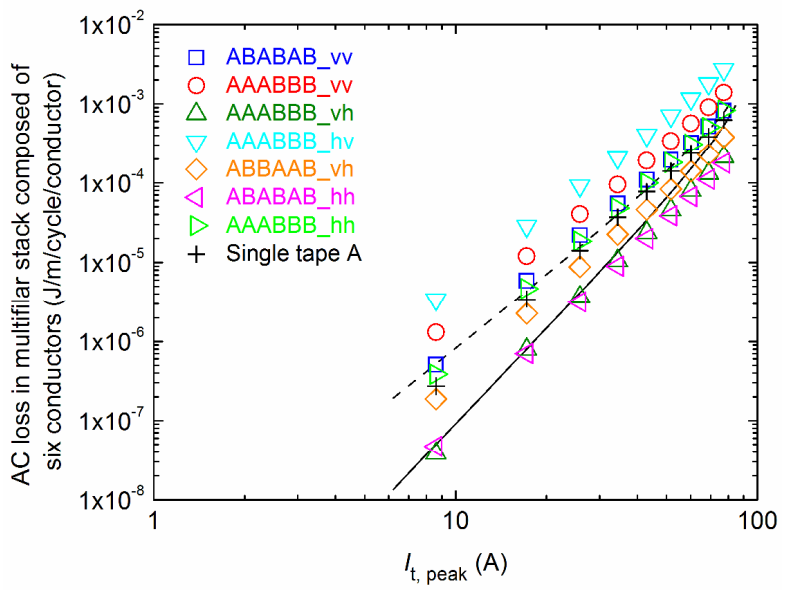

Fig. 14. Comparison of calculated AC losses in multifilar stacks composed of six conductors (MFS6) at $54 \mathrm{~Hz}$.

1) At first we compare AC loss values in the MFS6 with only one layer, i.e. ABABAB_vv and AAABBB_vv. AC loss values follow $Q_{\text {single }}<Q_{\text {ABABAB_vv }_{-}}<Q_{\text {AAABBB_vv }_{-} \text {, at any current }}$ amplitude. In other words, AC loss in the MFS6 with only one layer exceeds $Q_{\text {single }}$, due to strengthening of perpendicular magnetic field components in some of the gaps. The largest superposition of perpendicular magnetic field components occurs in the middle gap between the two upper conductors and the two lower conductors. At At $I_{\mathrm{t} \text {, peak }}=43 \mathrm{~A}\left(I_{\mathrm{t} \text {, peak }} / I_{\mathrm{c} 0}=0.5\right)$, $Q_{\text {ABABAB_vv }_{\text {vv }}}$ is $57.7 \%$ of $Q_{\text {AAABBB_vv }_{\text {vv }}}$ and $141.5 \%$ of $Q_{\text {single }}$.

2) When the conductors of MFS6 are configured in two layers, one possible configuration was considered, AAABBB_vh. At $I_{\mathrm{t} \text {, peak }}=43 \mathrm{~A}\left(I_{\mathrm{t}}\right.$, peak $\left./ I_{\mathrm{c} 0}=0.5\right), Q_{\text {AAABBB_vh }}$ is $2.37 \times 10^{-5} \mathrm{~J} / \mathrm{m} /$ cycle/conductor.

3) When the conductors of MSC6 are configured in three layers, they follow $Q_{\text {ABBAAB_vh }_{-}}<Q_{\text {single }}<Q_{\text {AAABBB_hv. }}$ At $I_{\text {t, peak }}$ $=43 \mathrm{~A}\left(I_{\mathrm{t}}\right.$, peak $\left./ I_{\mathrm{c} 0}=0.5\right), Q_{\text {ABBAAB_vh }_{-}}$accounts for $11.4 \%$ of $Q_{\text {AAABBB_hv }}, 59.7 \%$ of $Q_{\text {single. }}$.

4) When the conductors of MFS6 are configured in six layers side by side, they follow $Q_{\text {ABABAB_hh }_{-}}<Q_{\text {single }}<Q_{\text {AAABBB_hh }_{-}}$. 


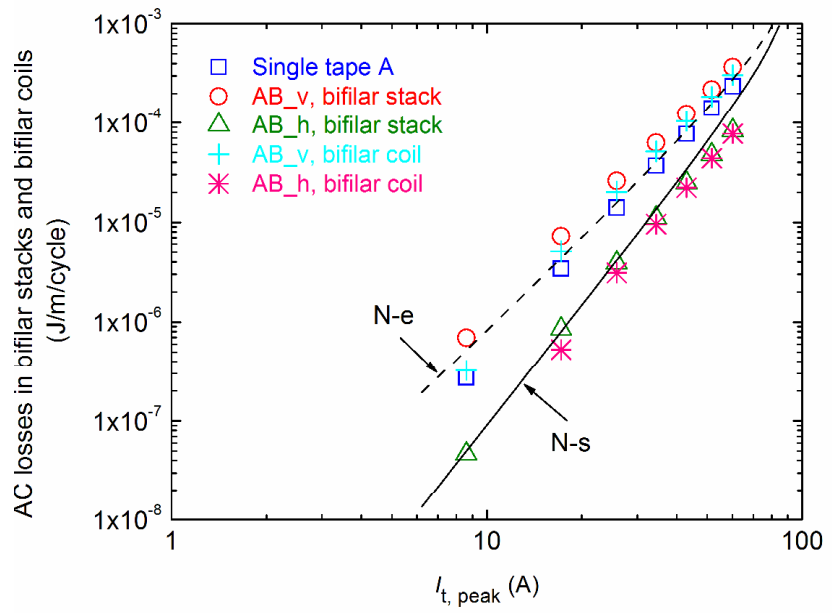

Fig. 15. AC loss comparison in bifilar stacks and 8-turn bifilar coils, plotted against transport current, at $54 \mathrm{~Hz}$.

At $I_{\mathrm{t}, \text { peak }}=43 \mathrm{~A}\left(I_{\mathrm{t}, \text { peak }} / I_{\mathrm{c} 0}=0.5\right), Q_{\mathrm{ABABAB} \_ \text {hh }}$ accounts for $20.1 \%$ of $Q_{\text {AAABBB hh, }} 25.9 \%$ of $Q_{\text {single }}$.

Comparing the AC loss performance in the seven multifilar stacks, $Q_{\text {ABABAB_hh, }} Q_{\text {AAABBB_vh }}$ agree well from one another, and also exhibit the lowest loss values in the seven stacks. On the other hand, $Q_{\mathrm{AAABBB}}$ hv has the highest AC loss compared to the other stacks.

\section{AC losses in solenoidal non-inductive coils}

Fig. 15 presents the AC loss results in bifilar stacks and 8turn solenoidal bifilar coils, calculated at $54 \mathrm{~Hz}$. It is worthwhile to note that the solenoidal bifilar coils are wound by the corresponding bifilar stacks with 8 turns in a helical structure. AC loss generated in non-inductive coil is noted as $Q_{\mathrm{AB} \_ \text {h, coil, }}$ and $Q_{\mathrm{AB} \_ \text {v, coil }}$ to distinguish the loss dissipated in each stack, $Q_{\mathrm{AB}_{-} \mathrm{h}}$ and $Q_{\mathrm{AB}_{-} \mathrm{v}}$.

It is clear that with the increase of transport current, $\mathrm{AC}$ loss value in bifilar stacks and bifilar coils increases. As shown in Fig. $15, Q_{\mathrm{AB} \_ \text {h, coil }}$ is much lower than $Q_{\mathrm{AB}_{-} \text {, coil }}$, at any transport current level, as the same tendency noticed in Fig. 5 for bifilar stack. At $I_{\mathrm{t} \text {, peak }}=43 \mathrm{~A}\left(I_{\mathrm{t} \text {, peak }} / I_{\mathrm{c} 0}=0.5\right), Q_{\mathrm{AB} \_ \text {h, coil }}$ is $21.4 \%$ of $Q_{\mathrm{AB} \_ \text {v. }}$ This shows one 8-turn bifilar coil wound by AB-h could reduce $\mathrm{AC}$ loss by around $80 \%$, compared to the bifilar coil wound by AB-v. It is also observed that $Q_{\mathrm{AB}_{-} \text {, coil }}$ is slightly lower than $Q_{\mathrm{AB}_{-} \mathrm{h}}$, and $Q_{\mathrm{AB}_{-} \mathrm{v} \text {, coil }}$ is smaller than $Q_{\mathrm{AB}_{-} \mathrm{v}}$. This is due to better perpendicular magnetic field cancellation gained by multiple turns in the coil.

Fig. 16(a) and 16(b) show the magnetic field distribution in the multifilar coils wound by the stack of $\mathrm{AB}_{-} \mathrm{v}$ and $\mathrm{AB} \mathrm{B}_{-} \mathrm{h}$, respectively, at the peak current when $I_{\mathrm{t} \text {, peak }} / I_{\mathrm{c} 0}=0.5$. The two coils are noted as $\mathrm{MFC}_{\mathrm{AB}_{-} \mathrm{v}}$ and $\mathrm{MFC}_{\mathrm{AB}_{-} \mathrm{h}}$. Only four turns in half model was shown here considering the symmetry of the coil. There is strong perpendicular magnetic field distributed around each conductor edge of $\mathrm{MFC}_{\mathrm{AB}_{-} \mathrm{v}}$, as compared to $\mathrm{MFC}_{\mathrm{AB}_{-} \mathrm{h}}$. This phenomenon determines the $\mathrm{AC}$ loss in $\mathrm{MFC}_{\mathrm{AB}_{-} \mathrm{h}}$ and $\mathrm{MFC}_{\mathrm{AB}_{-} \mathrm{v}}$. There is almost even magnetic field distribution in every turn of $\mathrm{MFC}_{\mathrm{AB}_{-} \mathrm{h}}$. On the other hands, stronger field was observed in the end turn of $\mathrm{MFC}_{\mathrm{AB}} \mathrm{v}$, and weaker field distribution in middle turn of $\mathrm{MFC}_{\mathrm{AB}_{-} \mathrm{v}}$.

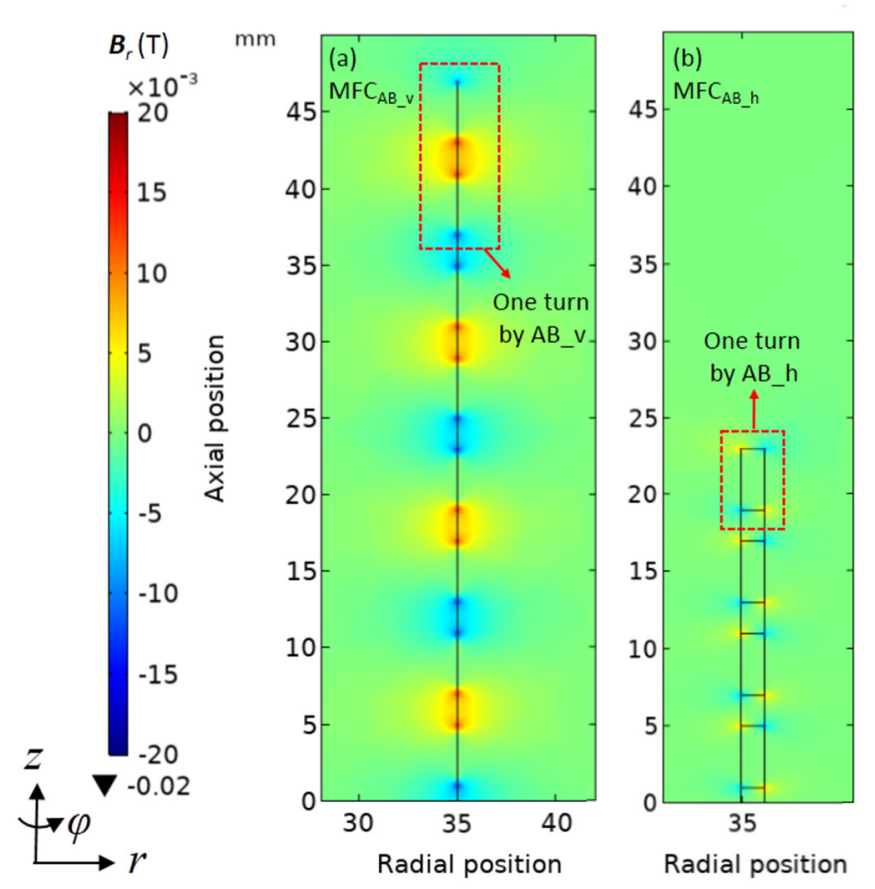

Fig. 16. Perpendicular magnetic field distribution in 8-turn multifilar coils wound by stack AB_v and AB_h, at the peak current when $I_{\mathrm{t}, \text { peak }} / I_{\mathrm{c} 0}=0.5$. Only half of the model was shown here due to symmetry.

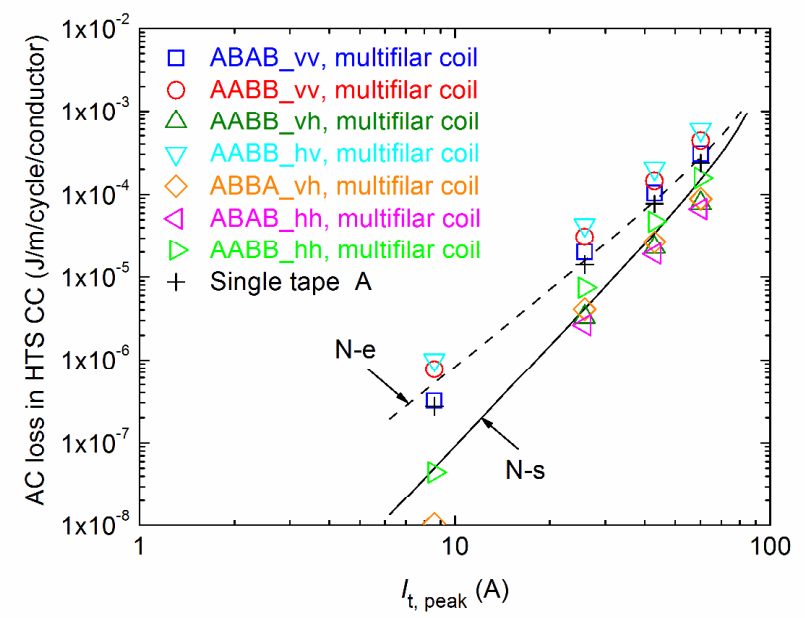

Fig. 17. AC loss comparison 8-turn multifilar coils wound by MFS4 with different configurations, plotted against transport current, at $54 \mathrm{~Hz}$.

This also indicates the configuration of AB_v does not lead to uniform radial magnetic field cancellation in coil turns at different positions. On the contrary, the configuration of $A B \_h$ results to uniform and thorough radial field cancellation.

Fig. 17 shows the AC loss results in 8-turn solenoidal multifilar coils wound by MFS4 with different configurations, compared with AC loss in single tape at $54 \mathrm{~Hz}$. It is apparent that AC loss in multifilar coils increases with increasing the amplitude of transport current. AC loss in the multifilar coil wound by $\mathrm{ABAB} \_$hh, $Q_{\mathrm{ABAB}}$ hh, coil is the lowest, and $\mathrm{AC}$ loss in

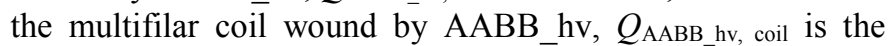
highest. This is consistent with the trend that $Q_{\mathrm{ABAB} \_ \text {hh }}$ is the lowest and $Q_{\text {AABB_hv }}$ is the highest, among the stacks. AC loss tendency in multifilar coils agrees well with that shown in 
multifialr stacks. At $I_{\mathrm{t}, \text { peak }}=43 \mathrm{~A}\left(I_{\mathrm{t}, \text { peak }} / I_{\mathrm{c} 0}=0.5\right), Q_{\mathrm{ABAB} \_ \text {hh, coil }}$ is $24.7 \%$ of $Q_{\text {single. }}$. This shows one 8 -turn multifilar coil wound by ABAB-hh could reduce AC loss by around $75.3 \%$, compared to that in single tape.

\section{DISCUSSIONS}

Assembling all conductors of non-inductive stack into one layer has simple structure, i.e. arrange all conductors vertically "head to head", but it will not lead to uniform and effective cancellation of the perpendicular magnetic field component. In this case, AC loss per conductor, in the non-inductive stack is still higher than that in single wire. This conclusion was proven from the fact that $Q_{\mathrm{AB}_{-} \mathrm{v}}>Q_{\text {single }}, Q_{\mathrm{AABB}_{-} \mathrm{vv}}>Q_{\mathrm{ABAB}_{-} \mathrm{vv}}>Q_{\text {single }}$, and $Q_{\text {AAABBB_vv }_{\text {vv }}}>Q_{\text {ABABAB_vv }_{\text {vv }}}>Q_{\text {single. }}$.

The most complicated structure for non-inductive stack was achieved by assembling all conductors horizontally "side by side", since this configuration consists the highest layer number, and it brings difficulty in coil bobbin fabrication and coil winding process. However, the lowest AC loss value could be achieved in such configuration. It was proven by the fact that $Q_{\mathrm{AB}_{-} \mathrm{h}}, Q_{\mathrm{ABAB} \_\mathrm{h}}$, and $Q_{\mathrm{ABABAB} \_\mathrm{h}}$ has the lowest $\mathrm{AC}$ loss amplitude in each scenarios.

The configurations for non-inductive stack, $Q_{\mathrm{AB}}$ h, $Q_{\mathrm{AABB}}$ vh and $Q_{\mathrm{AAABBB} \text { _vh }}$, are preferred with simple structure and low $\mathrm{AC}$ loss comparable to the smallest value. In these configuration, all conductors will be assembled into two layers, half conductors carrying current in one direction were arranged vertically "head to head" in one layer, and the other half conductors carrying current in opposite direction were arranged vertically "head to head" into the second layer, whilst aligned with the first layer. This was confirmed by the low AC loss value in $Q_{\mathrm{AB} \_ \text {h, }} Q_{\mathrm{AABB} \_ \text {vh }}$ and $Q_{\mathrm{AAABBB} \text { vh }}$.

\section{CONCLUSION}

Non-inductive coils wound by simple stacks with multiple conductors in parallel is a promising technology to achieve high current carrying capability for solenoidal SFCL coil and low $\mathrm{AC}$ loss. There are different ways to configure the conductors of non-inductive stack and hence, different configuration will lead to various AC loss and layer number. The more layer numbers, the more complex coil structure and its manufacture.

To optimize the electromagnetic field distribution and reduce AC loss for solenoid SFCL, different configurations of noninductive solenoidal coils wound with stacks were proposed and analyzed systematically. Numerical calculations were carried out to investigate the magnetic distribution and AC loss for each configuration of non-inductive stack. 8-turn noninductive solenoidal coils wound by single wire and two parallel conductors with seven possible configurations were simulated.

$\mathrm{AC}$ loss in non-inductive coil wound by parallel conductors is dependent on the configuration of parallel conductors. AC loss in the configured non-inductive coil with multiple turns is slightly lower than the loss value in the same configured noninductive stack, due to a better magnetic field cancellation effect.
Single layer configurations of non-inductive stack do not have uniform perpendicular magnetic field cancellation, leading to high AC losses. Multiple layer configurations where all conductors are arranged horizontally "side by side" offers the lowest $\mathrm{AC}$ loss but the most complex structure. Two layer configurations, where half conductors carrying current in one direction while arranged vertically "head to head" in one layer, and the other half conductors carrying current in opposite direction while arranged vertically "head to head" into the second layer, are preferred for practical application as they are structurally easy to fabricate and have low AC losses.

\section{REFERENCES}

[1] M. Noe, and M. Steurer, "High-temperature superconductor fault current limiters: concepts, applications, and development status," Supercond. Sci. Technol., vol. 20, no. 3, R15, 2007.

[2] Y. Xin, "Review on superconducting fault current limiters," South. Power Syst. Technol., vol. 9, pp. 1-9, 2015.

[3] S. H. Lim, H. S. Choi, D. C. Chung, Y. H. Jeong, Y. H. Han, T. H. Sung, and B. S. Han, "Fault current limiting Characteristics of resistive type SFCL using a transformer," IEEE Trans. Appl. Supercond., vol. 15, no. 2, pp. 2055-2058, June 2005.

[4] A. Morandi, and S. Imparato, "A DC-operating resistive-type superconducting fault current limiter for AC applications," Supercond. Sci. Technol., vol. 22, no. 4, 2009, Art. no. 045002.

[5] X. Pei, X. Zeng, A. C. Smith and D. Malkin, "Resistive superconducting fault current limiter coil design using multistrand MgB2 wire," IEEE Trans. Appl. Supercond., vol. 25, no. 3, pp. 1-5, June 2015, Art. no. 5602105.

[6] M. Yazdani-Asrami, M. Staines, G. Sidorov, M. Davies, J. Bailey, N. Allpress, and S. A. Gholamian, "Fault current limiting HTS transformer with extended fault withstand time," Supercond. Sci. Technol., vol. 32, Art. no. 035006, 2018.

[7] L. Ying, L, J. Sheng, B. Lin, L. Yao, J. Zhang, Z. Jin, Y. Li, and Z. Hong, "AC loss and contact resistance of resistive type fault current limiter using YBCO coated conductors," IEEE Trans. Appl. Supercond., vol. 22, no. 3, pp. 5602204-5602204, 2011

[8] B. Shen, C. Li, J. Geng, Q. Dong, J. Ma, J. Gawith, K. Zhang, J. Yang, X. Li, Z. Huang, and T. A. Coombs, "Investigation on power dissipation in the saturated iron-core superconducting fault current limiter," IEEE Trans. Appl. Supercond., vol. 29, no. 2, pp. 1-5. 2018.

[9] D. K. Park, J. S. Bang, S. E. Yang, T. K. Ko, Y. S. Yoon, M. C. Ahn, and K. Sim, "Theoretical and experimental analysis of AC loss characteristic of bifilar pancake coil with coated conductor," IEEE Trans. Appl. Supercond., vol. 18, no. 2, pp. 1232-1235, 2008.

[10] P. Tixador. Superconducting Fault Current Limiter: Innovation for The Electric Grids (Vol. 3), World Scientific. 2018.

[11] W. Song, X. Pei, J. Xi and X. Zeng, "A novel helical superconducting fault current limiter for electric propulsion aircraft," IEEE Trans. Transp. Electrification, doi: 10.1109/TTE.2020.2998417.

[12] H. Alafnan, X. Zeng, X. Pei, M. Khedr, M. Zhang, and W. Yuan, "Analysing faults and SFCL response in electric aircraft," In 14th European Conference on Applied Superconductivity (EUCAS 2019), 2020.

[13] J. W. Zhuang, X. F. Zhang, F. Yang, and C. Wang, "Analysis and design of fault current limiter for ship DC power system," Proc. Chinese Society of Electrical Engineering, vol. 25, no. 20, 2005.

[14] C. Lee, H. Kang, M. C. Ahn, T. K. Ko, and B. Y. Seok, "Short-circuit test of a novel solenoid type high-Tc superconducting fault current limiter," Cryogenics, vol. 47, no. 7-8, pp. 380-386, 2007.

[15] X. Pei, "Superconducting fault current limiter with integrated vacuum interrupter," Doctoral dissertation, University of Manchester, United Kingdom, 2012.

[16] M. Yazdani-Asrami, M. Staines, G. Sidorov, and A. Eicher, "Heat transfer and recovery performance enhancement of metal and superconducting tapes under high current pulses for improving fault current-limiting behavior of HTS transformers," Supercond. Sci. Technol., vol. 33, no. 9, Art. no. 095014, 2020.

[17] J. Šouc, F. Gömöry, and M. Vojenčiak, "Coated conductor arrangement for reduced $\mathrm{AC}$ losses in a resistive-type superconducting fault current 
limiter," Supercond. Sci. Technol., vol. 25, no. 1, Art. no. 014005, 2011.

[18] Y. Wang, M. Zhang, F. Grilli, Z. Zhu, and W. Yuan, "Study of the magnetization loss of CORC® cables using a 3D TA formulation," Supercond. Sci. Technol., vol. 32, no. 2, 2019, Art. no. 025003 .

[19] M. Vojenčiak, A. Kario, B. Ringsdorf, R. Nast, D. C. van der Laan, J. Scheiter, B. Rucntsch, F. Gömöry, and W. Goldacker, "Magnetization ac loss reduction in HTS CORC $($ cables made of striated coated conductors," Supercond. Sci. Technol., vol. 28, no. 10, 2015. Art. no. 104006.

[20] R. A. Badcock, N. J. Long, M. Mulholland, S. Hellmann, A. Wright and K. A. Hamilton, "Progress in the manufacture of long length YBCO Roebel cables," IEEE Trans. Appl. Supercond., vol. 19, no. 3, pp. 32443247, June 2009.

[21] N. J. Long, R. Badcock, P. Beck, M. Mulholl, N. Ross, M. Staines, and R. G. Buckley, "Narrow strand YBCO Roebel cable for lowered AC loss," J. Phys. : Conf. Series, vol. 97, no. 1, 2008. Art. no.. 012280.

[22] Z. Jiang, N. Amemiya, K. Kakimoto,Y. Iijima, T. Saitoh, and Y. Shiohara, "The dependence of AC loss characteristics on the space in stacked YBCO conductors," Supercond. Sci. Technol., vol. 21, no. 1, 2007, Art. no. 015020

[23] W. Song, Z. Jiang, X. Zhang, M. Staines, C. W. Bumby, R. A. Badcock, and J. Fang, "Transport AC loss measurements in bifilar stacks composed of YBCO coated conductors," IEEE Trans. Appl. Supercond., vol. 28, no. 4, pp. 1-6, June 2018, Art no. 5900606.

[24] B. Shen, J. Li, J. Geng, L. Fu, X. Zhang, H. Zhang, and T. A. Coombs, "Investigation of AC losses in horizontally parallel HTS tapes," Supercond. Sci. Technol., vol. 30, no. 7, Art. no. 075006, 2017.

[25] H. Kang, C. Lee, K. Nam, Y. S. Yoon, H. M. Chang, T. K. Ko, and B. Y. Seok, "Development of a $13.2 \mathrm{kV} / 630$ A (8.3 MVA) high temperature superconducting fault current limiter," IEEE Trans. Appl. Supercond., vol. 18 , no. 2, pp. 628-631, 2008 .

[26] S. Dai, T. Ma, C. Xue, L. Zhao, Y. Huang, L. Hu, B. Wang, T. Zhang, X. $\mathrm{Xu}$, L. Cai, and H. Chen, "Development and test of a $220 \mathrm{kV} / 1.5 \mathrm{kA}$ resistive type superconducting fault current limiter," Physica C, vol. 565, Art no. 1253501, 2019.

[27] M. Noe, A. Hobl, P. Tixador, L. Martini and B. Dutoit, "Conceptual Design of a $24 \mathrm{kV}, 1 \mathrm{kA}$ Resistive Superconducting Fault Current Limiter," IEEE Trans. Appl. Supercond., vol. 22, no. 3, pp. 56003045600304, June 2012, Art no. 5600304.

[28] J. Bock, A. Hobl, J. Schramm, S. Krämer and C. Jänke, "Resistive superconducting fault current limiters are becoming a mature technology," IEEE Trans. Appl. Supercond., vol. 25, no. 3, pp. 1-4, June 2015, Art. no. 5600604.

[29] W. Song, J. Fang and Z. Jiang, "Numerical AC loss analysis in HTS stack carrying nonsinusoidal transport current," IEEE Trans. Appl. Supercond., vol. 29, no. 2, pp. 1-5, March 2019, Art. no. 5900405.

[30] Z. Hong, A. M. Campbell, and T. A. Coombs, "Numerical solution of critical state in superconductivity by finite element software," Supercond. Sci. Technol., vol. 19, pp. 1246-1252, 2006.

[31] F. Grilli, R. Brambilla, F. Sirois, A. Stenvall, S. Memiaghe, "Development of a three-dimensional finite-element model for hightemperature superconductors based on the $\mathrm{H}$-formulation," Cryogenics, vol. 53, pp. 142-147, 2013.

[32] M. D. Ainslie, T. J. Flack, and A. M. Campbell, "Calculating transport AC losses in stacks of high temperature superconductor coated conductors with magnetic substrates using FEM," Physica C, vol. 472, no. 1, pp. 50-56, 2012.

[33] F. Grilli, E. Pardo, A. Stenvall, D. N. Nguyen, W. Yuan, and F. Gömöry, "Computation of losses in HTS under the action of varying magnetic fields and currents," IEEE Trans. Appl. Supercond., vol. 24, no. 1, pp. 78-110, 2013

[34] W. Song, Z. Jiang, M. Staines, R. A. Badcock, and J. Fang, "Experimental and numerical transport AC losses in a four-strand Roebel cable bifilar stack," Supercond. Sci. Technol., vol. 31, no. 11, Art. no. 115001, 2018.

[35] M. Yazdani-Asrami, S. Asghar Gholamian, S. M. Mirimani, J. Adabi, "Calculation of AC Magnetizing loss of ReBCO superconducting tapes subjected to applied distorted magnetic fields," J. Supercond. Nov. Magn., vol. 31, no. 12, pp. 3875-3888, 2018

[36] B. Shen, F. Grilli, and T. A. Coombs, "Overview of H-Formulation: A Versatile Tool for Modelling Electromagnetics in High Temperature Superconductor Applications", IEEE Access, vol. 8, 2020.

[37] B. Shen, F. Grilli, and T. A. Coombs, "Review of the AC Loss Computation for HTS using H formulation", Superconductor Science and Technology, vol. 33, no. 3, 2020.

[38] D. N. Nguyen, F. Grilli, S. P. Ashworth, and J. O. Willis, "AC loss study of antiparallel connected YBCO coated conductors," Super-cond. Sci. and Technol., vol. 22, 2009, Art. no. 055014.

[39] F. Gömöry, M. Vojenčiak, E. Pardo, M. Solovyov, and J. Šouc, "AC losses in coated conductors," Supercond. Sci. Technol., vol. 23, no. 3, 2010, Art. No. 034012.

[40] Y. B. Kim, C. F. Hempstead, A. R. Strnad, "Critical persistent currents in hard superconductors," Phys. Rev. Lett., vol. 9, pp. 306-312, 1962.

[41] N. M. Strickland and S. C. Wimbush, "The magnetic-field dependence of critical current: what we really need to know," IEEE Trans. Appl. Supercond., vol. 27, 2017, Art. no. 8000505 .

[42] S. Li, D.-X. Chen, Y. Fan, and J. Fang, "Transport ac loss in a rectangular thin strip with power-law E (J) relation," Physica C, vol. 508, pp. 12-16, 2015.

[43] M. Zhang, J. Kvitkovic,S. V. Pamidi, and T. A. Coombs, "Experimental and numerical study of a YBCO pancake coil with a magnetic substrate," Supercond. Sci. Technol., vol. 25, no. 12, Art. no. 125020, 2012.

[44] B. Shen, C. Li, J. Geng, X. Zhang, J. Gawith, J. Ma, Y. Liu, F. Grilli and T. A. Coombs, "Power dissipation in HTS coated conductor coils under the simultaneous action of AC and DC currents and fields", Superconductor Science and Technology, vol. 31, no. 7, 2018.

[45] W. Song, Z. Jiang, X. Zhang, M. Staines, R. A. Badcock, J. Fang, Y. Sogabe, and N. Amemiya, "AC loss simulation in a HTS 3-Phase 1 MVA transformer using H formulation," Cryogenics, vol. 94, pp. 14-21, 2018.

[46] W. T. Norris, "Calculation of hysteresis losses in hard superconductor carrying AC: Isolated conductors and edges of thin sheets," J. Phys. D: Appl. Phys., vol. 3, pp. 489-507, 1970.

[47] Z. Jiang, W. Zhou, Q. Li, M.Yao, J. Fang, N. Amemiya, and C. W. Bumby, "The dynamic resistance of YBCO coated conductor wire: Effect of DC current magnitude and applied field orientation," Supercond. Sci. Technol., vol. 31, no. 3, Art. no. 035002, 2018. 